\title{
Knockdown of interferon-induced transmembrane protein 1 (IFITM1) inhibits proliferation, migration, and invasion of glioma cells
}

\author{
Fang Yu $\cdot$ Samuel S. M. Ng $\cdot$ Billy K. C. Chow $\cdot$ \\ Johnny Sze · Gang Lu • Wai Sang Poon • \\ Hsiang-Fu Kung $\cdot$ Marie C. M. Lin
}

Received: 19 May 2010/Accepted: 19 August 2010/Published online: 14 September 2010

(C) The Author(s) 2010. This article is published with open access at Springerlink.com

\begin{abstract}
Interferon-induced transmembrane protein 1 (IFITM1) has recently been identified as a new molecular marker in human colorectal cancer. However, its role in glioma carcinogenesis is not known. In this study, we demonstrated that suppression of IFITM1 expression significantly inhibited proliferation of glioma cells in a timedependent manner. The growth inhibitory effect was mediated by cell cycle arrest. Furthermore, IFITM1 knockdown significantly inhibited migration and invasion of glioma cells, which could be attributed to decreased expression and enzymatic activity of matrix metalloproteinase 9. Taken together, these results suggest that IFITM1 is a potential therapeutic target for gliomas.
\end{abstract}

Keywords IFITM1 - Glioma - Antiproliferation · Anti-invasion $\cdot$ Cell cycle

F. $\mathrm{Yu} \cdot \mathrm{G} . \mathrm{Lu} \cdot$ W. S. Poon - M. C. M. Lin

Brain Tumour Center, The Chinese University of Hong Kong,

Shatin, Hong Kong, China

e-mail: yufang@hkusua.hku.hk

G. $\mathrm{Lu}$

e-mail: lugang@surgery.cuhk.edu.hk

W. S. Poon

e-mail:wpoon@surgery.cuhk.edu.hk

G. Lu · W. S. Poon · M. C. M. Lin ( $\square)$

Division of Neurosurgery, Department of Surgery, The Chinese

University of Hong Kong, Shatin, Hong Kong, China

e-mail: mcllin@hkusua.hku.hk

F. Yu

Department of Pathology, Sir Run Run Shaw Hospital, School of Medicine, Zhejiang University, Hangzhou, China

\section{Introduction}

Glioma, derived from malignant glial cells, is the most frequent tumor in the central nervous system. According to its histopathological characteristics and clinical prognosis, it is classified into four grades. Glioblastoma multiforme (GBM), the grade IV glioma, is the most malignant type and accounts for $60 \%$ of all brain tumor cases [1]. Glioma cells are notorious for their rapid proliferation and widespread invasion to surrounding normal brain tissues. Despite recent advances in understanding the molecular mechanism involved in glioma carcinogenesis, it remains an untractable disease. Five-year survival in glioma is the lowest among all tumor types, and median survival of GBM is only 10-12 months [2, 3]. A major breakthrough in glioma treatment clearly requires the discovery of novel target-based molecules based on our improved understanding of the molecular pathogenesis of glioma.

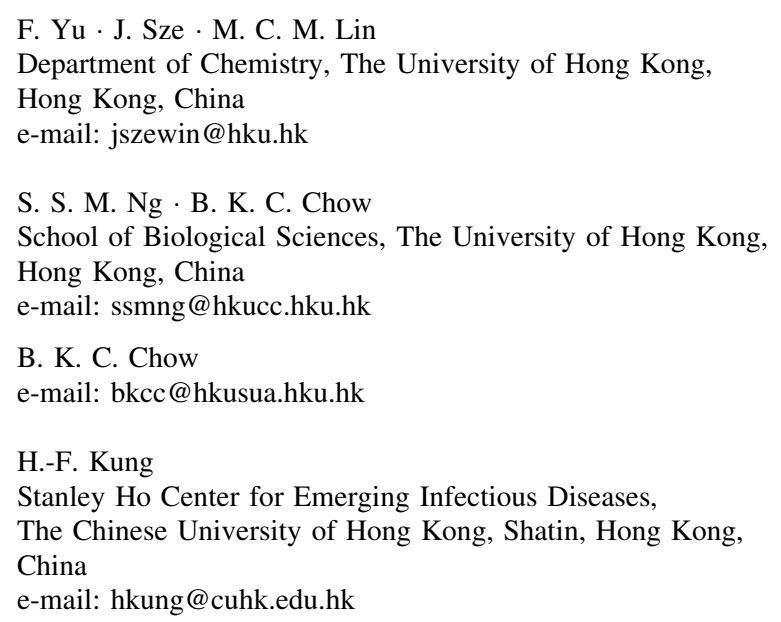


Interferon-induced transmembrane protein 1 (IFITM1), also known as 9-27, CD225, and Leu13, is a member of the interferon-induced transmembrane protein family. IFITM1 is a component in the membrane complex that transduces antiproliferative and homotypic adhesion signals in lymphocytes [4, 5]. It is also responsible for mouse primordial germ cell development [6, 7]. Furthermore, it has been shown to promote cancer progression by enhancing cell migration and invasion in gastric cancer and head and neck cancer [8, 9]. Recently, Seyfried et al. identified several novel brain tumor membrane biomarkers using liquid chromatography coupled with tandem mass spectrometry. Among the 25 differentially regulated membrane proteins, expression of IFITM1 was found to be significantly higher in CT-2A astrocytoma cells than in terminally differentiated mouse astrocytes [10], suggesting that it might be involved in the neoplasia process of gliomas. In the present study, therefore, we examined the oncogenic roles of IFITM1 in human glioma cells.

\section{Materials and methods}

\section{Cell culture and reagents}

Human high-grade glioma cell lines (U-87 MG, U-373 MG, U-138 MG, SW1088) were obtained from the American Type Culture Collection (ATCC, Manassas, VA). Cell line LN-308 was a gift from Dr. M.E. Hegi (University Hospital Lausanne, Switzerland). The cells were maintained in minimum essential medium (MEM; Invitrogen; for U-87 MG, U-373 MG, U-138 MG, and LN-308) or Leibovitz's L-15 medium (Invitrogen, for SW1088) supplemented with $10 \%$ fetal bovine serum (FBS; Invitrogen) under $5 \% \mathrm{CO}_{2}$ at $37^{\circ} \mathrm{C}$.

Small interfering RNA (siRNA) duplex oligonucleotides specifically targeting human IFITM1 messenger RNA (mRNA) (silFITM1; 5'-AACTCATGACCATTGGATTC A-3') or firefly luciferase mRNA (siLuc; 5'-AACGTAC GCGGAATACTTCGA-3') were synthesized and purified by Sigma-Proligo (The Woodlands, TX, USA). All antibodies used in this study were bought from Santa Cruz Biotechnology (Santa Cruz, CA).

Semiquantitative reverse-transcription polymerase chain reaction (RT-PCR)

Total RNA was extracted from the five glioma cell lines with the use of RNeasy mini kit (Qiagen). First-strand complementary DNA (cDNA) was reverse-transcribed by SuperScript First-Strand cDNA System (Invitrogen) and was amplified by PCR. The PCR conditions were set up as follows: $45 \mathrm{~s}$ at $95^{\circ} \mathrm{C}, 45 \mathrm{~s}$ at $55^{\circ} \mathrm{C}$, and $1 \mathrm{~min}$ at $72^{\circ} \mathrm{C}$ for
30 cycles (for IFITM1) or for 22 cycles [for glyceraldehyde 3-phosphate dehydrogenase (GAPDH)].

\section{3-(4,5-Dimethylthiazol-2-yl)-2,5-diphenyltetrazolium} bromide (MTT) assay

One day before transfection, $3 \times 10^{4} \mathrm{U}-373 \mathrm{MG}$ or U-87 MG cells in $100 \mu$ l growth medium were each seeded onto 96-well cell culture plate. The cells were transfected with siIFITM1 or siLuc at final concentration of $100 \mathrm{nM}$ and oligofectamine transfection reagent (Invitrogen). Cells incubated with opti-MEM and transfection reagent were used as a negative control (mock transfection). At 24, 48 or $72 \mathrm{~h}$ after transfection, $10 \mu \mathrm{l}$ sterile MTT dye $(5 \mathrm{mg} / \mathrm{ml}$; Sigma, St. Louis, MO) was added to each well. After $4 \mathrm{~h}$ incubation at $37^{\circ} \mathrm{C}$, the MTT solution was replaced with $100 \mu$ dimethyl sulfoxide (DMSO). Spectrometric absorbance at $570 \mathrm{~nm}$ was measured with the use of a microplate reader (Spectra Max 340, Molecular Devices, Sunnyvale, CA).

Wound healing assay

Equal numbers $\left(1.5 \times 10^{5}\right)$ of U-373 MG or U-87 MG cells transfected by $100 \mathrm{nM}$ siIFITM1 or siLuc for $24 \mathrm{~h}$ were treated with $0.25 \%$ trypsin-ethylenediamine tetraacetic acid (EDTA; Invitrogen) and plated onto a 48-well plate at $1 \times 10^{5}$ cells per well. When $90 \%$ confluence was reached, a single wound was created by gently scratching the attached cells using a sterile plastic pipette tip. Debris was removed by washing the cells with serum-free medium. Immediately or $24 \mathrm{~h}$ after incubation at $37^{\circ} \mathrm{C}$, phase-contrast images were photographed digitally. The cells that migrated to the wounded area, or those with extended protrusion from the borders of the wound, were counted from three randomly selected areas per well. The experiment was performed for three times in triplicates.

In vitro invasion assay

Equal numbers $\left(3 \times 10^{4}\right)$ of U-373 MG or U-87 MG cells transfected by $100 \mathrm{nM}$ siIFITM1 or siLuc for $24 \mathrm{~h}$ were plated onto a 24-well cell culture insert coated with $\mathrm{Ma}$ trigel with $8-\mu \mathrm{m}$ pores (Becton Dickinson Labware, Bedford, MA). MEM with 10\% FBS was added to the lower chamber as chemoattractant. After incubation for $22 \mathrm{~h}$, the cells on the upper surface of the Matrigel membrane were removed. The penetrated cells on the lower surface of the membrane were fixed with $100 \%$ methanol, stained with $1 \%$ toluidine (Sigma), and quantified in five randomly selected areas per insert. The experiment was performed for three times in triplicates. 
Cell cycle analysis

One day before transfection, $1.5 \times 10^{5} \mathrm{U}-373 \mathrm{MG}$ or U-87 MG cells were seeded onto six-well cell culture plates. The cells were transfected with siIFITM1 or siLuc at final concentration of $100 \mathrm{nM}$ using oligofectamine transfection reagent (Invitrogen) for $72 \mathrm{~h}$. The transfected cells were fixed in ice-cold 70\% ethanol and stained by Coulter DNAPrep Reagents Kit (Beckman Coulter, Fullerton, CA). Cellular DNA content of $5 \times 10^{5}$ cells from each sample was determined with the use of an EPICS ALTRA flow cytometer (Beckman Coulter). Cell cycle phase distributions were analyzed by ModFit LT 2.0 software (Verify Software House, Topsham, ME) using the data obtained from three separate experiments in which each transfection was done in triplicate.

\section{Real-time RT-PCR}

Total RNA was extracted from U-373 MG cells transfected by siIFITM1 or siLuc for $72 \mathrm{~h}$ using RNeasy mini kit (Qiagen). First-strand cDNA was reverse-transcribed from $1 \mu \mathrm{g}$ total RNA using the Super-Script First-Strand cDNA System (Invitrogen) and amplified by Platinum SYBR Green qPCR SuperMix-UDG (Invitrogen). A master mix was prepared for each PCR reaction, which included Platinum SYBR Green qPCR SuperMix-UDG, forward primer, reverse primer, and $10 \mathrm{ng}$ template cDNA. PCR conditions were $2 \mathrm{~min}$ at $50^{\circ} \mathrm{C}$ and $10 \mathrm{~min}$ at $95^{\circ} \mathrm{C}$, followed by 40 cycles of $95^{\circ} \mathrm{C}$ for $15 \mathrm{~s}, 58^{\circ} \mathrm{C}$ for $60 \mathrm{~s}$, and $95^{\circ} \mathrm{C}$ for $15 \mathrm{~s}$. The data was analyzed by calculating the $C_{\mathrm{t}}$ values by the use of the formula $2-\left(B_{1}-A_{1}\right)$, where $B_{1}$ is the $C_{\mathrm{t}}$ value of the amplified genes and $A_{1}$ is the $C_{\mathrm{t}}$ value of GAPDH.

\section{Western blotting}

For Western blotting analysis, U-373 MG and U-87 MG cells transfected by siIFITM1 or siLuc for 24,48 , and $72 \mathrm{~h}$ were washed twice with ice-cold phosphate-buffered saline (PBS) and lysed for $30 \mathrm{~min}$ in a radioimmunoprecipitation assay lysis buffer [50 mM Tris- $\mathrm{HCl}, 1 \% \mathrm{NP}-40,0.5 \%$ sodium deoxycholate, $150 \mathrm{mM}$ sodium chloride, $0.1 \%$ sodium dodecyl sulfate (SDS)] containing $1 \mathrm{mM}$ phenylmethylsulfonyl fluoride and protease inhibitor cocktail (Sigma). The lysates were centrifuged at $14,000 \times g$ for $20 \mathrm{~min}$, and the supernatants were removed. The protein concentrations of the supernatants were determined by using a bicinchoninic acid protein assay kit (Pierce, Rockford, IL). Heat-denatured protein samples $(40 \mu \mathrm{g}$ per lane) were resolved by SDS-polyacrylamide gel electrophoresis
(PAGE) (4\% stacking gel and $12 \%$ separating gel) and transferred to nitrocellulose membranes (Amersham Biosciences, Piscataway, NJ). The membranes were incubated with $5 \%$ milk for $2 \mathrm{~h}$ to block nonspecific binding, followed by incubation with a primary goat antibody against human IFITM1, or primary mouse antibodies against human cyclin D1, cyclin-dependent kinase 2 (CDK2), cyclindependent kinase inhibitor $1 \mathrm{~B}\left(\mathrm{p} 27^{\mathrm{kip} 1}\right)$, matrix metalloproteinase 9 (MMP9), cyclin B1, cyclin-dependent kinase 1 (CDK1), and $\beta$-actin, respectively. The membranes were washed three times for $30 \mathrm{~min}$ in Tris-buffered saline (TBS) with $0.1 \%$ Tween 20 and then incubated with the corresponding secondary antibodies. The membranes were washed thoroughly in TBS with $0.1 \%$ Tween 20 and the bound antibodies were detected with enhanced chemiluminescence detection reagents (Amersham Bioscience, Piscataway, NJ) according to the manufacturer's instructions. Band intensity was quantified with the use of ImageQuant software (Molecular Dynamics, Sunnyvale, CA).

\section{Gelatin zymography}

U-373 MG cells transfected by siIFITM1 or siLuc for $48 \mathrm{~h}$ were first washed twice with serum-free medium and then cultured with the same medium for additional $24 \mathrm{~h}$. The medium was collected and clarified by centrifugation to remove cells and debris. The supernatants were removed, and the protein concentrations of the supernatants were determined by using a bicinchoninic acid protein assay kit (Pierce, Rockford, IL). Samples were prepared by mixing the supernatants with an equal volume of $2 \times$ nonreducing loading buffer for $15 \mathrm{~min}$ at room temperature. Samples (15 $\mu \mathrm{g}$ per lane) were resolved by $10 \%$ polyacrylamide gel containing $1 \mathrm{mg} / \mathrm{ml}$ gelatin. After electrophoresis, the gel was washed twice in $2.5 \%$ Triton $\mathrm{X}-100$ for $30 \mathrm{~min}$ at room temperature. The gel was then incubated with developing buffer $(50 \mathrm{mM}$ Tris- $\mathrm{HCl}, \mathrm{pH} 7.4 ; 10 \mathrm{mmol} / \mathrm{l}$ $\mathrm{CaCl}_{2}$ ) overnight at room temperature, stained with Coomassie Brilliant Blue $(0.25 \% \mathrm{w} / \mathrm{v})$, and then destained in methanol:acetic acid:water solution (45:10:45). A clear zone indicates the presence of gelatinolytic activity in zymography.

\section{Statistical analysis}

All experiments were performed three times in triplicates. The data were analyzed by Student's $t$ test (Prism 3.0, GraphPad Software, San Diego, CA) and are expressed as mean \pm standard deviation (SD). Differences were considered statistically significant at $P$ value $<0.05$. 


\section{Results}

Expression of IFITM1 in human glioma cell lines

mRNA and protein levels of IFITM1 in five human glioma cell lines (U-87 MG, U-373 MG, U-138 MG, SW1088, and LN-308; grades II-IV) were analyzed by RT-PCR and Western blotting, respectively. IFITM1 was expressed in all five glioma cell lines, and IFITM1 protein levels were generally consistent with mRNA levels (Fig. 1). According to data from ATCC (http://www.atcc.org/), the four cell lines with higher IFITM1 expression are all tumorigenic in nude mice. By contrast, U-138 MG, which displayed the lowest level of IFITM1 level, was the only nontumorigenic cell line tested.

Effect of IFITM1 knockdown on the growth of glioma cells

To elucidate the functional role of IFITM1 in glioma carcinogenesis, we examined the effect of IFITM1 mRNA knockdown on glioma cell growth in vitro by transfecting U-373 MG or U-87 MG cells with siIFITM1 (which specifically targets IFITM1 mRNA), siLuc (which targets an unrelated firefly luciferase mRNA) or transfection reagent alone (mock transfection). U-373 MG and U-87 MG cell lines were chosen as our cell model, because they both showed a high level of IFITM1 expression and are widely used tumorigenic cell lines in glioma study. Our Western blotting data showed that the inhibitory effect of siIFITM1 on IFITM1 expression was time dependent and most effective at $72 \mathrm{~h}$ after transfection in U-373 MG or U-87 MG cells (Fig. 2a). Thus, $72 \mathrm{~h}$ was chosen as the time point for siIFITM1 transfection in subsequent experiments.

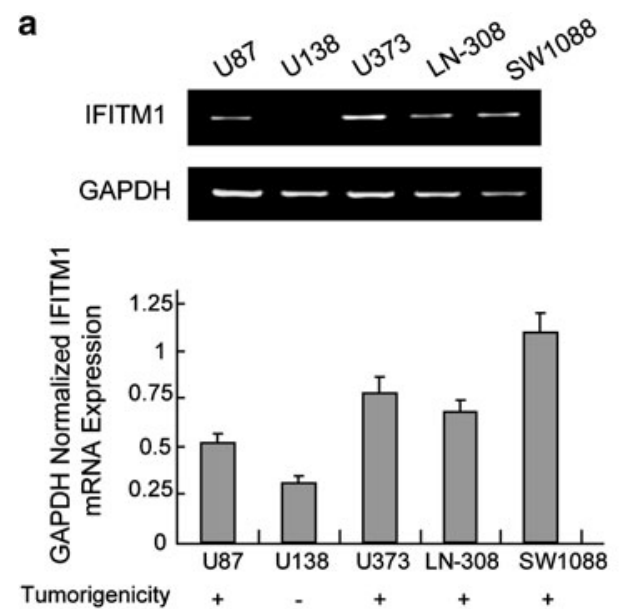

Fig. 1 Expression of IFITM1 in five human glioma cell lines. a Representative agarose gel pictures showing expression of IFITM1 in five glioma cell lines as measured by semiquantitative RT-PCR. The bar graph shows GAPDH-normalized IFITM mRNA expression in those cell lines. b Western blotting analysis showing protein levels
We next determined the numbers of viable cells at various time intervals after siIFITM1 or siLuc transfection, using MTT assays. Transfection with siIFITM1, but not siLuc, statistically significantly inhibited growth of U-373 MG and U-87 MG cells in a time-dependent manner (Fig. 2b).

Effect of IFITM1 knockdown on cell migration and invasion in vitro in glioma cells

To examine the effect of IFITM1 knockdown on glioma cell migration, wound healing assay was performed using U-373 MG or U-87 MG cells transfected with siIFITM1 or siLuc. Immediately or $24 \mathrm{~h}$ after incubation, the cells that migrated to the wounded area, or those with extended protrusion from the borders of the wound, were counted from three randomly selected areas per well. Our data showed that siIFITM1 transfection markedly inhibited migration of U-373 MG and U-87 MG cells (Fig. 3a). To examine the effect of IFITM1 knockdown on glioma cell invasion, in vitro invasion assay was performed. After incubation for $22 \mathrm{~h}$, the penetrated cells on the lower surface of the membrane were quantified in five randomly selected areas per insert. Our data showed that transfection with siIFITM1 significantly suppressed invasion of U-373 MG and U-87 MG cells (Fig. 3b).

Effect of IFITM1 knockdown on cell cycle progression in glioma cells

To better understand the mechanism of siIFITM1-induced cell growth inhibition, we performed flow cytometry to examine the cell cycle profiles of U-373 MG cells or U-87 MG cells transfected with siIFITM1 or siLuc. The

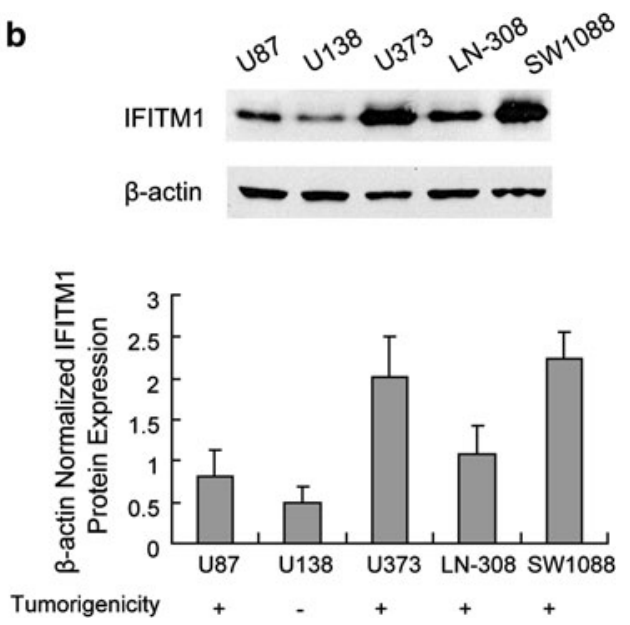

of IFITM1 in five glioma cell lines. The bar graph shows $\beta$-actinnormalized IFITM protein expression in those cell lines. The intensities of the results were quantified by densitometry and ImageQuant software (molecular dynamics). Tumorigenic and nontumorigenic glioma cell lines are labeled "+" and "-", respectively 
Fig. 2 Effect of IFITM1 knockdown on glioma cell growth. a Western blotting analysis showing the timedependent effect of siIFITM1 transfection on levels of IFITM1 and $\beta$-actin expression in U-373 MG cells (left) and U-87 MG cells (right). The bar graph shows relative IFITM1 protein expression levels expressed as percentage of IFITM1 expression in control cells transfected with siLuc.

$* P<0.05$. b MTT assays showing the time-dependent effect of siIFITM1 transfection on U-373 MG cells (left) and U-87 MG cells (right). $\mathrm{OD}_{570 \mathrm{~nm}}$ values obtained at $24 \mathrm{~h}$ post transfection were normalized as 1 . $* P<0.001$ a
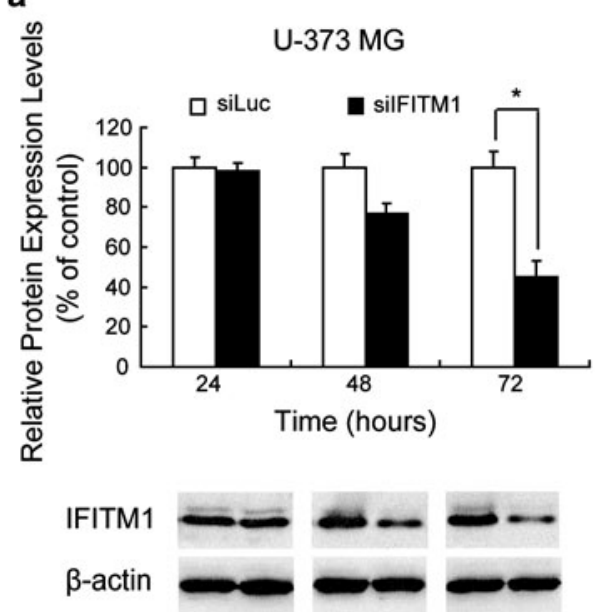

b

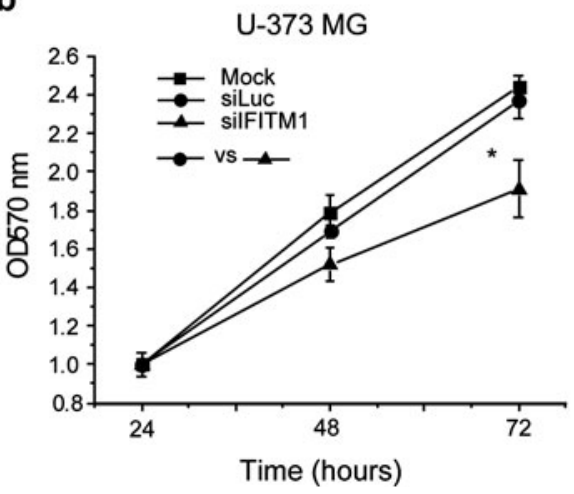

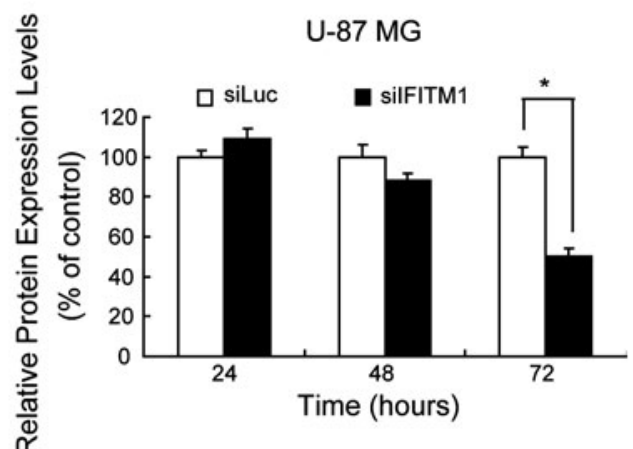

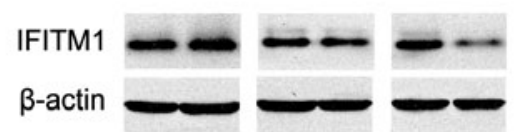

U-87 MG

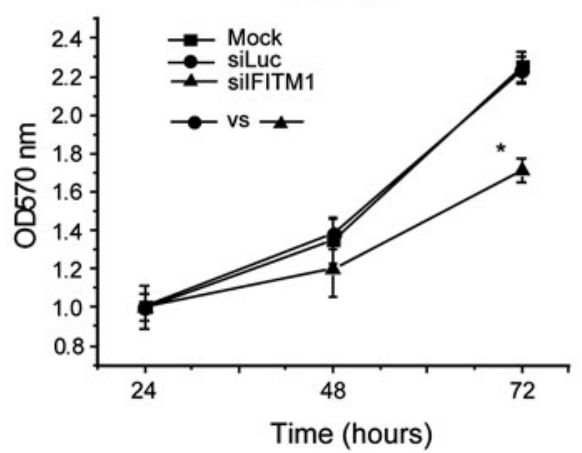

siIFITM1-transfected U-373 MG cells had a higher number of cells at G0-G1 phase and a lower number of cells at S and G2-M phases. On the other hand, the siIFITM1-transfected U-87 MG cells had a higher population of cells at G2-M phase (Fig. 4), suggesting that the effect of growth inhibition induced by siIFITM1 in glioma cells was mediated by cell cycle arrest at either G0G1 or G2-M phase.

Effect of IFITM1 knockdown on expression of cellcycle-related genes and expression and activity of matrix metalloproteinase 9 (MMP9) in glioma cells

To investigate the signaling of IFITM1-induced cell cycle arrest and cell invasion inhibition, we examined the effect of IFITM1 suppression on well-documented genes related to cell cycle control (cyclin A, cyclin B1, cyclin D1, cyclin E, $C D K 2, C D K 4, p 21$, and $p 27^{k i p 1}$ ) and cell invasion [MMP2, $M M P 9$, tissue inhibitor of metalloproteinase-1 (TIMP1), and TIMP2] by real-time RT-PCR in U-373 MG cells transfected with siIFITM1. The relative mRNA levels (normalized by mRNA level of GAPDH) of cyclin A, cyclin B1, cyclin D1, cyclin E, CDK2, CDK4, and MMP9 were significantly reduced in siIFITM1-transfected cells at $72 \mathrm{~h}$ after transfection (the mean fold changes of the genes were $-1.8 \pm 0.15,-2.5 \pm 0.43,-4.9 \pm 0.6, \quad-1.5 \pm 0.28$, $-1.7 \pm 1.0,-1.5 \pm 0.5$, and $-4.6 \pm 0.15$, respectively). On the contrary, the relative mRNA level of $p 27^{k i p 1}$ was significantly increased, with mean fold change of $1.6 \pm 0.2$ (Fig. 5a).

We next examined protein levels of cyclin D1, CDK2, and $\mathrm{p} 27^{\mathrm{kip} 1}$ in $\mathrm{U}-373 \mathrm{MG}$ cells at $72 \mathrm{~h}$ after siIFITM1 or siLuc transfection. Consistent with the real-time RT-PCR results, the relative protein levels (normalized by $\beta$-actin protein expression) of cyclin D1 and CDK2 were reduced, whereas the relative protein level of $\mathrm{p} 27^{\mathrm{kip} 1}$ was increased by siIFITM1 transfection (Fig. 5b).

The inhibitory effect of IFITM1 on cell cycle progression was different in U-373 MG cells and U-87 MG cells. To explain the discrepancy, we also examined the protein levels of cyclin D1, CDK2, p2 $7^{\mathrm{kip} 1}$, cyclin B1, and CDK1 in U-87 MG cells at $72 \mathrm{~h}$ after siIFITM1 or siLuc transfection. Our results showed that the relative protein levels (normalized by $\beta$-actin protein expression) of cyclin D1 and $\mathrm{p} 27^{\mathrm{kip} 1}$ were increased, whereas the relative protein levels of CDK1 was reduced by siIFITM1 transfection (Fig. 5c).

Finally, the protein level and enzymatic activity of MMP9 were measured at $72 \mathrm{~h}$ after transfection by 
a

a
U-373 MG

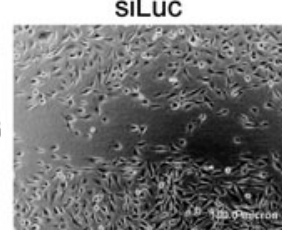

silFITM1
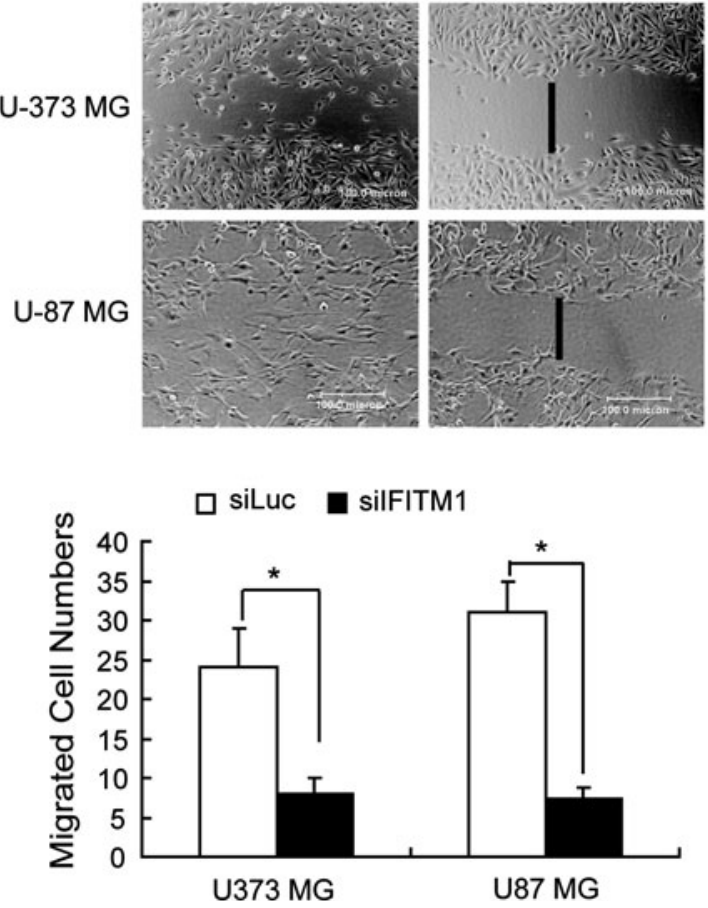

Fig. 3 Effect of IFITM1 knockdown on glioma cell migration and invasion in vitro. a Wound healing assays showing the inhibitory effect of siIFITM1 on migration of U-373 MG and U-87 MG cells. The bar graph shows mean migrated cells at $24 \mathrm{~h}$ after wound
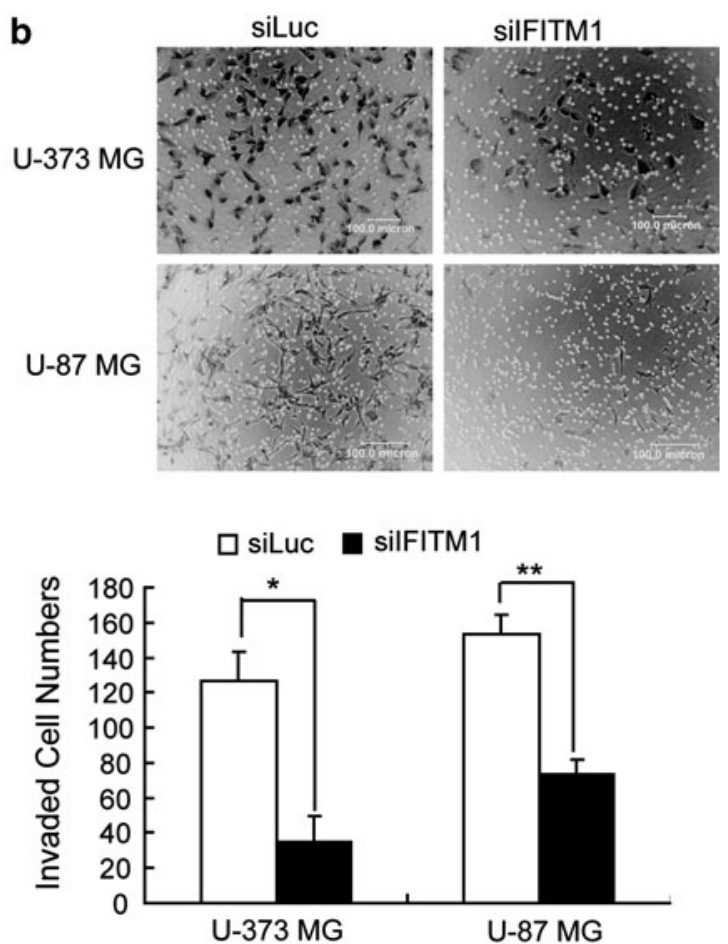

formation. $* P<0.01$. b In vitro Matrigel assays showing the inhibitory effect of silFITM1 on invasion of U-373 MG and U-87 MG cells. The bar graph indicates the mean number of invaded cells at $22 \mathrm{~h}$ after cell seeding. $* P<0.01$; $* * P=0.0031$
Fig. 4 Effect of siIFITM1 on cell cycle progression of glioma cells. U-373 MG cells (left) or U-87 MG cells (right) transfected with siIFITM1 or siLuc were collected $72 \mathrm{~h}$ post transfection and subjected to flow cytometry analysis. Data are presented as mean $\pm \mathrm{SD}$ from three independent experiments. $* P<0.01$, ** $P=0.0015$
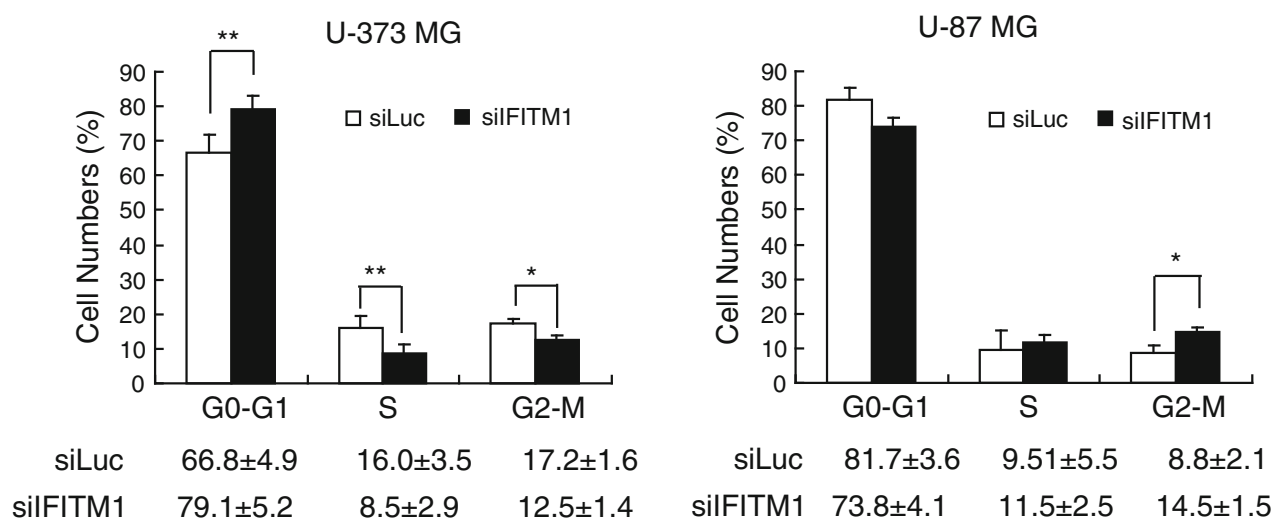

Western blotting and gelatin zymography, respectively. The relative protein level of MMP9 in siIFITM1-transfected cells was significantly lower than that in siLuctransfected U-373 MG cells. The gelatin zymography assay also detected a weaker lytic zone at the molecular mass corresponding to MMP9 in the siIFITM1-transfected cells (Fig. 5d).

\section{Discussion}

The role of IFITM1 in carcinogenesis is unclear. Nevertheless, recent IFITM1 expression data in different cancer types have led us to hypothesize that it might possess oncogenic function; for instance, IFITM1 expression has been found to be greatly elevated in colon cancer [11], cervical cancer [12], esophageal cancer [13], ovarian cancer, and mouse astrocytoma [10, 14]. In this study, we determined IFITM1 expression levels in five human glioma cell lines and investigated its roles in proliferation, migration, and invasion glioma cells. Our results showed that IFITM1 was expressed in the five human glioma cell lines, and its expressions were positively correlated with their tumorigenicity. Suppression of IFITM1 by siIFITM1 transfection inhibited proliferation, migration, and invasion of U-373 MG and U-87 MG glioma cells. Together, these 
Fig. 5 Effect of IFITM1 knockdown on expression of cell-cycle-related genes and expression and activity of MMP in glioma cells. a Real-time RT-PCR showing the effect of siIFITM1 on mRNA levels of genes related to cell cycle control (cyclin A, cyclin B1, cyclin D1, cyclin E, CDK2, $C D K 4, p 21$, and $\left.p 27^{k i p l}\right)$ and cell invasion (MMP2, MMP9, TIMP1, and TIMP2). Dashed horizontal lines indicate relative mRNA levels normalized by GAPDH mRNA expression at \pm 1.5 -fold. Expression levels below -1.5 -fold were regarded as decreased expression, whereas those above 1.5 -fold were regarded as increased expression. b Western blotting analysis showing the effect of siIFITM1 on expression levels of IFITM1, cyclin D1, CDK2, $\mathrm{p} 27^{\mathrm{kip} 1}$, and $\beta$-actin in U-373

MG cells. The bar graph shows relative IFITM1, cyclin D1, $\mathrm{CDK} 2$, and $\mathrm{p} 27^{\mathrm{kip} 1}$ protein levels in siIFITM1-transfected cells versus those in control siLuc-transfected cells.

$* P=0.033$, ** $P<0.01$.

c Western blotting analysis showing the effect of siIFITM1 on expression levels of IFITM1, cyclin D1, CDK2, p27 $7^{\mathrm{kip} 1}$, cyclin $\mathrm{B} 1, \mathrm{CDK} 1$, and $\beta$-actin in U-87 MG cells. The bar graph shows relative IFITM1, cyclin D1, CDK2, p27 ${ }^{\mathrm{kip} 1}$, cyclin $\mathrm{B} 1$, and CDK1 protein levels in siIFITM1-transfected cells versus those in control siLuc-transfected cells.

$* P=0.006$, ** $P<0.05$.

d Western blotting analysis and gelatin zymography assays showing the effect of siIFITM1 on MMP9 expression and enzymatic activity in U-373 MG cells. The bar graph shows the relative integrated densities of the bands in siIFITM1transfected cells expressed as percentage values of those in control siLuc-transfected cells. * $P=0.0095, * * P<0.001$ a

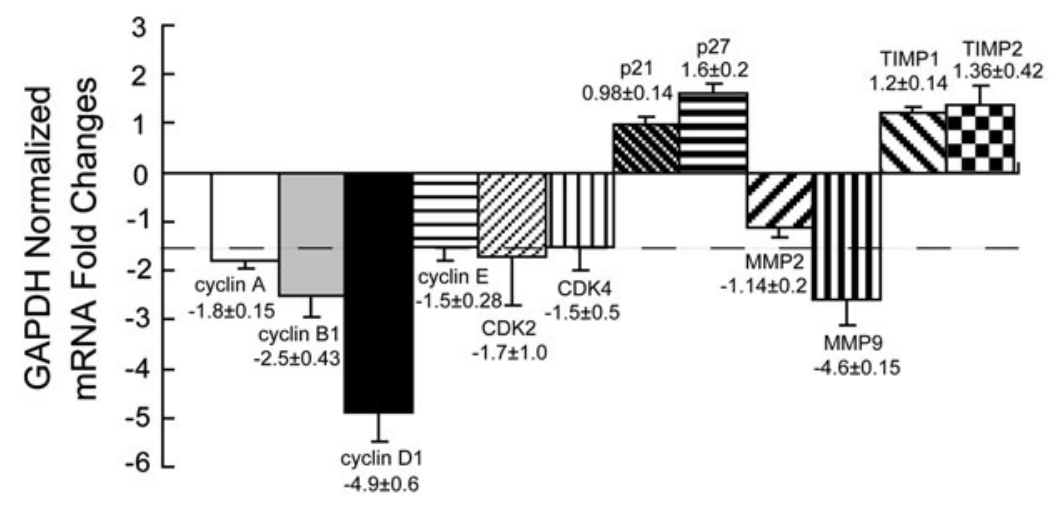

b siluc SilFITM1
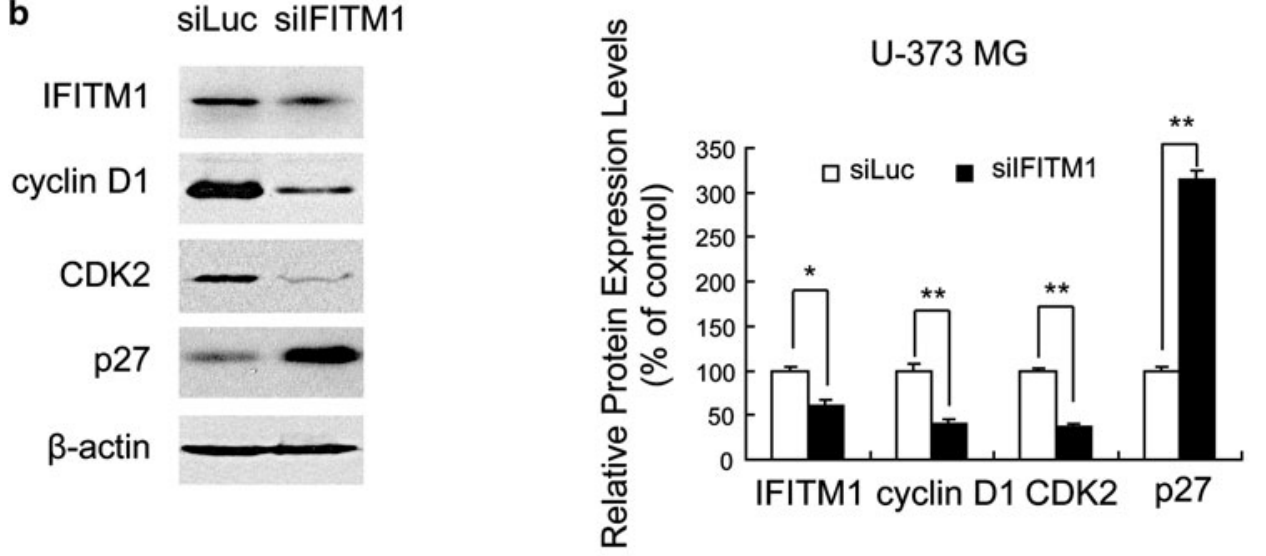

c siluc SilFITM1

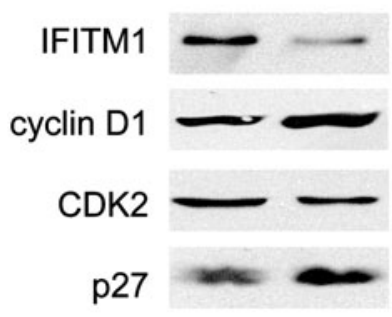

cyclin B1

CDK1

$\beta$-actin

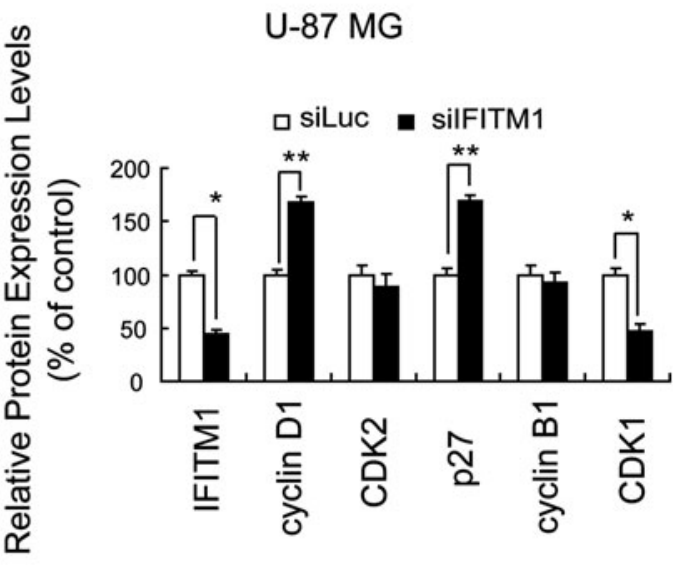

d

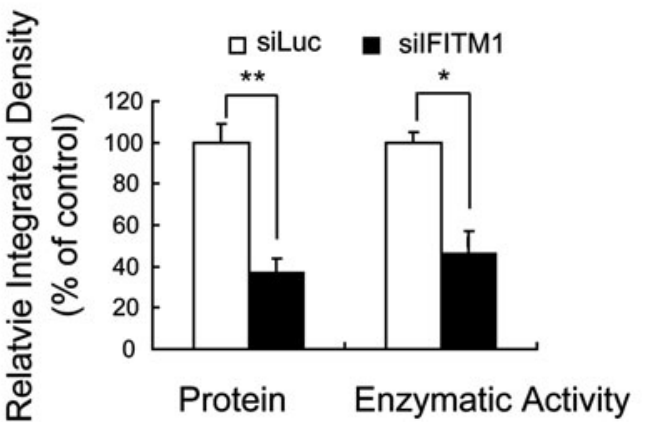


findings suggest for the first time that IFITM1 might be a potential candidate oncogene in glioma.

Our flow cytometry data showed that the growth inhibitory effect of siIFITM1 was mediated by cell cycle arrest. Of note, the siIFITM1-transfected U-373 MG cells displayed G0-G1 phase arrest, which could be mediated via decreased expression of G1/S-specific cyclin D1 and CDK2, and increased expression of $\mathrm{p} 27^{\mathrm{kip} 1}$ (Fig. 5b). By contrast, the siIFITM1-transfected U-87 MG cells showed G2-M phase arrest, which could be mediated by increased expression of cyclin D1 and p27 $7^{\mathrm{kip} 1}$, and decreased expression of CDK1. Furthermore, this discrepancy may be explained by the fact that U-373 MG cells express mutant p53, whereas U-87 MG cells possess wild-type p53 [15]. Because p53 is required for both G0-G1 and G2-M phase transitions [16], and the Gadd45-mediated G2-M arrest was wild-type p53-dependent [17], a significantly smaller number of siIFITM1transfected U-373 MG cells should be able to enter the S phase and progress to the G2-M phase. As a result, U-373 MG cells and U-87 MG cells arrested at different cell cycle checkpoints after siIFITM1 transfection.

Metastasis is one of the hallmarks of malignant cancers. Degradation of basement membranes and stromal extracellular matrix (ECM) is an initial step for metastasis. To this end, matrix metalloproteinases (MMPs) play a crucial role to degrade the physical barrier of ECM. Furthermore, MMPs are required to maintain a microenvironment for growth, invasion, and angiogenesis of tumor cells [18]. MMP9, a member of the MMP protein family, can degrade both denatured collagen and type IV collagen. MMP9 activity was also reported to be closely correlated with glioma progression [19]. Consistent with these findings, our results showed that IFITM1 knockdown significantly inhibited migration and invasion of glioma cells, and the inhibition was associated with reduced expression and activity of MMP9.

IFITM1 is commonly considered as a protein induced by interferon $\alpha$ and $\gamma$ [20,21], and it is involved in signal transduction of antiviral, anti-inflammation, and antiproliferation activities mediated by interferons in our immune system [22, 23]. However, with the improved understanding of the natural immune response to cancer, the roles of interferons in carcinogenesis are different from previously recognized. The relationship between the host immune system and cancer includes three phases, namely elimination, equilibrium, and escape. Interferons act as central coordinators between the interaction of the host immune system and cancer cells. At the elimination phase, interferon $\alpha$ and $\gamma$ are released by the host immune system in response to tumor-specific antigens and help to destroy nascent transformed cells. Tumor cells that are not killed at the first phase can then progress to the equilibrium phase. When the balance is broken by immune inhibition or emergence of tumor-cell variants, the escape phase begins, where the cancer cells escape the mechanisms that operate to suppress them and they become clinically detectable and grow progressively [24, 25]. In contrast to the elimination phase, the escape phase is poorly understood. Yang et al. [8] reported that gastric cancer cells overexpressing IFITM1 were more resistant to natural killer cells. Thus, we proposed that, under the influence of interferons at the escape phase, IFITM1 may be produced by glioma cells so as to antagonize the attack by the host immune system by promoting cell proliferation and invasion.

In summary, the data presented here provide the first evidence that IFITM1 is expressed in human glioma cell lines. Suppression of IFITM1 expression by siIFITM1 inhibited proliferation of glioma cells by arresting cell cycle progression. We further showed that siIFITM1 transfection inhibited migration and invasion of glioma cells by decreased expression and activity of MMP9. Therefore, our data suggest that IFITM1 is a potential therapeutic target for malignant gliomas.

Acknowledgments Conflicts of interest statement None declared.

Open Access This article is distributed under the terms of the Creative Commons Attribution Noncommercial License which permits any noncommercial use, distribution, and reproduction in any medium, provided the original author(s) and source are credited.

\section{References}

1. Ohgaki H, Kleihues P (2005) Epidemiology and etiology of gliomas. Acta Neuropathol 109:93-108. doi:10.1007/s00401-0050991-y

2. Maher EA, Furnari FB, Bachoo RM, Rowitch DH, Louis DM, Cavenee WK, DePinho RA (2001) Malignant glioma: genetics and biology of a grave matter. Gene Dev 15:1311-1333. doi: 10.1101/gad.891601

3. Stupp R, Mason WP, van den Bent MJ, Weller M, Fisher B, Taphoorn MJB et al (2005) Radiotherapy plus concomitant and adjuvant temozolomide for glioblastoma. New Engl J Med 352:987-996

4. Lewin AR, Reid LE, Mcmahon M, Stark GR, Kerr IM (1991) Molecular analysis of a human interferon-inducible gene family. Eur J Biochem 199:417-423. doi:10.1111/j.1432-1033.1991.tb16139.x

5. Sato S, Miller A, Howard M, Tedder T (1997) Regulation of B lymphocyte development and activation by the CD19/CD21/ CD81/Leu 13 complex requires the cytoplasmic domain of CD19. J Immunol 159:3278-3287

6. Tanaka SS, Yamaguchi YL, Tsoi B, Lickert H, Tam PPL (2005) IFITM/mil/fragilis family proteins IFITM1 and IFITM3 play distinct roles in mouse primordial germ cell homing and repulsion. Dev Cell 9:745-756. doi:10.1016/j.devcel.2005.10.010

7. Wylie C (2005) IFITM1-mediated cell repulsion controls the initial steps of germ cell migration in the mouse. Dev Cell 9:723-724. doi:10.1016/j.devcel.2005.11.009

8. Yang Y, Lee JH, Kim KY, Song HK, Kim JK, Yoon SR, Cho D, Song KS, Lee YH, Choi I (2005) The interferon-inducible 9-27 gene modulates the susceptibility to natural killer cells and the invasiveness of gastric cancer cells. Cancer Lett 221:191-200. doi:10.1016/j.canlet.2004.08.022 
9. Hatano H, Kudo Y, Ogawa I, Tsunematsu T, Kikuchi A, Abiko Y, Takata T (2008) IFN-induced transmembrane protein 1 promotes invasion at early stage of head and neck cancer progression. Clin Cancer Res 14:6097-6105. doi:10.1158/1078-0432. CCR-07-4761

10. Seyfried NT, Huysentruy LC, Atwood JA, Xia QW, Seyfried TN, Orlando R (2008) Up-regulation of NG2 proteoglycan and interferon-induced transmembrane proteins 1 and 3 in mouse astrocytoma: a membrane proteomics approach. Cancer Lett 263: 243-252. doi:10.1016/j.canlet.2008.01.007

11. Andreu P, Colnot S, Godard C, Laurent-Puig P, Lamarque D, Kahn A, Perret C, Romagnolo A (2006) Identification of the IFITM family as a new molecular marker in human colorectal tumors. Cancer Res 66:1949-1955. doi:10.1158/0008-5472. CAN-05-2731

12. Pan Z, Chen S, Pan X, Wang Z, Han H, Zheng W, Wang X, Li F, Qu S, Shao R (2010) Differential gene expression identified in Uigur women cervical squamous cell carcinoma by suppression subtractive hybridization. Neoplasma 57:123-128. doi:10.4149/ neo_2010_02_123

13. Chattopadhyay I, Phukan R, Singh A, Vasudevan M, Purkayastha J, Hewitt S, Kataki A, Mahanta J, Kapur S, Saxena S (2009) Molecular profiling to identify molecular mechanism in esophageal cancer with familial clustering. Oncol Rep 21:1135-1146. doi:10.3892/or_00000333

14. Gyorffy B, Dietel M, Fekete T, Lage H (2008) A snapshot of microarray-generated gene expression signatures associated with ovarian carcinoma. Int J Gynecol Cancer 18:1215-1233. doi: 10.1111/j.1525-1438.2007.01169.x

15. Gomez Manzano C, Fueyo J, Kyritsis AP, Steck PA, Roth JA, McDonnell TJ, Steck KD, Levin VA, Yung WKA (1996) Adenovirus-mediated transfer of the p53 gene produces rapid and generalized death of human glioma cells via apoptosis. Cancer Res 56:694-699
16. Levine AJ (1997) p53, the cellular gatekeeper for growth and division. Cell 88:323-331. doi:10.1016/s0092-8674(00)81871-1

17. Wang XW, Zhan QM, Coursen JD, Khan MA, Kontny HU, Yu LJ, Hollander MC, O'Connor PM, Fornace AJ, Harris CC (1999) GADD45 induction of a G(2)/M cell cycle checkpoint. Proc Natl Acad Sci USA 96:3706-3711

18. Nelson AR, Fingleton B, Rothenberg ML, Matrisian LM (2000) Matrix metalloproteinases: biologic activity and clinical implications. J Clin Oncol 18:1135-1149

19. Levicar N, Nutall RK, Lah TT (2003) Proteases in brain tumour progression. Acta Neurochir 145:825-838. doi:10.1007/s00701003-0097-z

20. Friedman RL, Manly SP, McMahon M, Kerr IM, Stark GR (1984) Transcriptional and posttranscriptional regulation of interferon-induced gene expression in human cells. Cell 38: 745-755. doi:10.1016/0092-8674(84)90270-8

21. Kelly J, Gilbert C, Stark G, Kerr I (1985) Differential regulation of interferon-induced mRNAs and c-myc mRNA by alpha- and gamma-interferons. Eur J Biochem 153:367-371. doi:10.1111/ j.1432-1033.1985.tb09312.x

22. Evans S, Lee D, Han T, Tomasi T, Evans R (1990) Monoclonal antibody to the interferon-inducible protein Leu-13 triggers aggregation and inhibits proliferation of leukemic B cells. Blood 76:2583-2593

23. Deblandre GA, Marinx OP, Evans SS, Majjaj S, Leo O, Caput D, Huez GA, Wathelet MG (1995) Expression cloning of an interferon-inducible $17-\mathrm{KDa}$ membrane-protein implicated in the control of cell-growth. J Biol Chem 270:23860-23866. doi: $10.1074 / j b c .270 .40 .23860$

24. Dunn GP, Koebel CM, Schreiber RD (2006) Interferons, immunity and cancer immunoediting. Nat Rev Immunol 6:836-848. doi:10.1038/nri1961

25. Dranoff G (2004) Cytokines in cancer pathogenesis and cancer therapy. Nat Rev Cancer 4:11-22. doi:10.1038/nrc1252 